\title{
Impact of Time-Management on the Student's Academic Performance: A Cross-Sectional Study
}

\author{
Adham Alyami, Abdulrahman Abdulwahed, Abdulrahman Azhar, Abdullah Binsaddik, \\ Saeed M. Bafaraj* \\ Department of Diagnostic Radiology, Faculty of Applied Medical Sciences, King Abdulaziz University, Jeddah, KSA \\ Email: *smbafaraj@kau.edu.sa
}

How to cite this paper: Alyami, A., Abdulwahed, A., Azhar, A., Binsaddik, A., \& Bafaraj, S. M. (2021). Impact of Time-Management on the Student's Academic Performance: A Cross-Sectional Study. Creative Education, 12, 471-485. https://doi.org/10.4236/ce.2021.123033

Received: January 26, 2021

Accepted: March 2, 2021

Published: March 5, 2021

Copyright ( 2021 by author(s) and Scientific Research Publishing Inc. This work is licensed under the Creative Commons Attribution International License (CC BY 4.0).

http://creativecommons.org/licenses/by/4.0/ (c) (i) Open Access

\begin{abstract}
For students to better manage their curriculum and achieve learning objectives, time management behaviors or skills are argued to improve the positive academic output. The aim of the study is to find the impact of time management on the academic performance of students among the diagnostic radiology technology students at KAU. This study had a cross-sectional survey-based study design. It was conducted among students of diagnostic radiology technology department at King Abdul-Aziz University from Sep. 2020 to Feb. 2021. For this study, 152 students were targeted among which 142 completed the questionnaire, making the response rate of $93.4 \%$. Among 142 participants, 75 (52.8\%) were females and $52(36.6 \%)$ were in the 2018 batch. Majority of the students i.e., 107 (75.4\%) had GPA between 4.5 - 5 in 2020. Whereas, $37.3 \%$ agreed or strongly agreed that they manage their time. Here, $69.2 \%$ students with 4.5 - 5 GPA strongly agreed that they meet their deadline ( $\mathrm{p}$ value $=0.005$ ) and $36.7 \%$ students with $4-4.5$ GPA strongly believed that their academic performance decreased due to mis planning ( $\mathrm{p}$ value $=0.005$ ). Around 71 (66.3\%) students with 4.5 - 5 GPA agreed or strongly agreed to make to do list or calendar ( $\mathrm{p}$ value 0.047 ). In conclusion, according to student's perception, preplanning their studies had been beneficial for their academic performance. However, less than half of the students agreed that they manage their time.
\end{abstract}

\section{Keywords}

Academic Achievement, Cross-Sectional, Radiology, Students, Time Management 


\section{Introduction}

Throughout the history, there has been great emphasis on the effective and efficient management of time, which has also been considered the key to success (Pugh \& Nathwani, 2017; Nasrullah \& Khan, 2015). Frederick Winslow Taylor gave the concept to use time management for analyzing time and motion studies of employees with aim to decrease time wasting and unproductive work (Savino, 2016). In this competitive era, for high performance, the organizations and directors emphasize on searching time management tools (Kumar \& Aithal, 2019). It has also been advised to start practicing time management from the early student life (Valle et al., 2016). Time management has also been defined as a form of self-management with a clear emphasis on time in understanding what activities to do; how to do them more efficiently; in what time it should be done and when is the correct time to the particular activity (Savino, 2016). It has also been association with low anxiety and greater academic achievement in students (Jenaabadi, Nastiezaie, \& Jalalzaei, 2016).

Whereas performing goal directed activities with the purpose of using time efficiently defines time management as "behavior" (Aeon \& Aguinis, 2017). The time management behavior has three basic surfaces that are: time attitudes, long-range planning and short-range planning (Aeon \& Aguinis, 2017). Time attitude is the positive or negative perspective towards the present, future and past time which had been directly linked to academic achievement, especially in scientific subjects (Nieuwoudt, \& Brickhill, 2017). The concept of handling everyday jobs for longer period of time and keeping up with the objectives set for significant dates is called the competence in long range planning. Whereas, planning in the short run for the day to a day activity or planning for tasks within a day or week is short-range planning. For students to better manage their curriculum and achieve learning objectives, these time management behaviors or skills are argued to improve the positive academic output (Razali et al., 2018).

There have been many studies that found association between greater academic achievement and effective time management as students acquire strategies that helps them in meeting competing demands (Nadinloyi et al., 2013; Kharadze, Gulua, \& Davit, 2017). The curriculums in higher education institutes are designed in a way that gets to peak and troughs in the workload of student requiring them to manage between their work-life balance, often without the support of institution. The non-cognitive personal behavior i.e., perspective of students regarding time management is also an effective predictor of educational achievement as with poor time management skills it gets difficult for students to plan their studies and which causes them anxiety and agitation at the assessment time which usually takes place at the end of the course (Scherer, Talley, \& Fife, 2017). Students have previously linked negative educational results with poor time management which could be partially self-serving bias, however, there have been ample studies that establish this association (Nadinloyi et al., 2013; Kha- 
radze, Gulua, \& Davit, 2017). There have been studies conducted on time management and educational achievement in business students (Sayari, Jalagat, \& Dalluay, 2017), nursing students (Nayak, 2019), management students (Gupta \& Chitkara, 2018), and engineering students (Adams \& Blair, 2019). However, none have been done on radiology students.

Previous studies have determined a positive effect of time management. Time management skills have indicated a positive effect on student learning and associated outcomes. A number of studies have identified the positive impact of time management. Time management skills have been shown to have a positive impact on student learning and student outcomes (Kearns \& Gardiner, 2007; Kelly, 2002; McKenzie, Gow, \& Schweitzer, 2004) and Krause \& Coates (2008) indicated that the ability for successfully managing their time is the benchmark of students in developing better study habits as well as strategies for success. Time management provides individuals the ways for structuring and controlling their activities (Claessens et al., 2004). In addition, report that the capacity to successfully manage their time is the foundation of students developing good study habits and strategies for success. Time management offers individuals the means to structure and control their activities (Claessens et al., 2004). In addition, Wang et al. (2011) have revealed that time management is essential beyond the university campus, where the ability to manage one's free time is revealed to substantially elevates the quality of life of an individual.

Time management is an immense distinction between web-based and formal education administratively and academically. There was the type of learning opportunities in formal education that integrates a clarification of everything (Nieuwoudt \& Brickhill, 2017). Teachers, in this system, are in front of students and they can ask anything regardless of any hesitation or delay. However, distance learning students have no such type of academic environment as compared to formal learners. The presence of physical distance is evidently reported between teacher and student in online learning (Ahmad \& Ch, 2017). Thereby, it is more complex for managing time and gaining high scores in distance education. Web-based students explore and learn online that just integrates incomplete data and the rest is their business for thinking about and examine further. It is an art for managing time and every learner must command and familiar on this skill for the sake of betterment (Broadbent, 2017). They experience in domestic as well as academic world because of this incompetence. In this regard, majority of learner's experience issues such as errand vulnerability and repugnance, so they commence for stalling their time administrative skills.

This study is based on existentialism philosophy, which is learner centered and offers direction to students about their study. Learners are self-responsible of their academics as they just seek guidance from their teachers, but take decisions as well as manage all activities themselves. This study is relied on pickle jar theory, which is the modern and relevant theory of time management (Sharma \& Would, 2004). This theory shows that human beings should construct balance among activities and things while integrating time management techniques ef- 
fectively. The success of students is determined through effective utilization of time at each phase in distance learning institutions and regular system.

Therefore, the aim of the study is to find the impact of time management on the academic performance of students among the diagnostic radiology technology students at KAU. The study objectives also include to find out the effectiveness of the preplanning and the instantaneous planning on academic performance. This research will add self-evaluation to student's daily habit and plan. Also, it will provide knowledge and recommendation for skills that can assist student for perfect personal strategic plan. It will also help to overcome the wrong common rumors statements about time management and will raise awareness about procrastinating behavior of time management and its effect on academic performance among radiology students.

\section{Methods}

This study had a cross-sectional survey-based study design. It was conducted among students of diagnostic radiology technology department at King Abdul-Aziz University. The study duration was six months from Sep 2020 to Feb 2021. Only the undergraduate students were included in the studies. Post-graduate students and students who had completed their education were excluded. The recommended sample size with $95 \%$ confidence level, 200 population size, $50 \%$ response distribution, and 5\% margin error were calculated to be 132 .

All registered undergraduate students were invited for taking part in the study. They were provided a brief overview of the project as well as information about confidentiality and research ethics. A total of 132 students responded by providing informed consent and completing the survey. The participation rate was just over $66 \%$ of the overall population. There might be some bias in the resultant data since the survey was self-developed. The nature of this bias is complex to establish, since the literature might recommend that both students should be responsive to complete this survey who have better time management skills and tend to procrastinate.

The academic performance of students such as GPA was acquired through the student administration system and departmental documents of the institution. Log files were also collected on students' participation in courses on the virtual learning platform. A unique random identifier was allocated to each student that would not show his or her identification to others beyond the research team. These unique codes enabled the combination of performance data, virtual learning platform files, and questionnaire responses in excel file, which did not comprise of any personally identifiable information. Once collected, such unique codes became irrelevant and were omitted.

This study was approved by the Research and ethics committee of faculty of applied Medical Science, KAU. The questionnaire was a self-designed, and had 22 questions on Likert scale of $1-5$ where 1 indicated strongly agree and 5 denoted strongly disagree and last question was about the GPA achieved in the last year. The questionnaire was prepared on the basis of self-management, aware- 
ness, management, preferences, and performance domains (Appendix 1).

Feedback was taken from research experts and changes were made before implementation. Also, an internal pilot study on 20 students was done before distributing it on the larger sample. The SPSS software version 25.0 was used to analyze the data. The Pearson chi-square test was conducted to find association between the time management and GPA achieved. The alpha coefficient was 0.05 and p-value below 0.05 was considered statistically significant.

\section{Results}

Among 142 participants, 75 (52.8\%) were females and 67 (47.2\%) were males. Around 52 (36.6\%) were in the 2018 batch, 45 (31.7\%) were in the 2019 batch and $45(31.7 \%)$ were in the 2020 batch of radiology. Figure 1 represents the GPA of student. Majority of the students i.e., 107 (75.4\%) had last year GPA between 4.5 - 5. Table 1 represents the frequency of the answers given by the participants for each question related to time management. Around 64.8\% disagreed or strongly disagreed that preplanning methods are inefficient, indicating that majority of the students perceive preplanning to be efficient for academic performance. Whereas, $37.3 \%$ agreed or strongly agreed that they manage their time. Majority of the students (92.3\%) agreed that they meet their deadlines (Table 1). Though, $74.6 \%$ agree or strongly agree that their time management skills can be approved.

Table 2 is showing the relationship between GPA of last year and student perception regarding time management. Here, 36.7\% students with 4 - 4.5 GPA strongly believed that their academic performance decreased due to mis planning ( $\mathrm{p}$ value $=0.005)$. Whereas, Table 2 shows the relationship between GPA of last year and time management behavior. Here, 69.2\% students with 4.5 - 5 GPA strongly agreed that they meet their deadline $(\mathrm{p}$ value $=0.005)$. Similarly, Table 3 showed the relationship between various factors and last year GPA. Here, majority i.e., $71(66.3 \%)$ students with 4.5 - 5 GPA agreed or strongly agreed to make to do list or calendar (p value 0.047) (Table 4).

Frequency of students with various GPAs

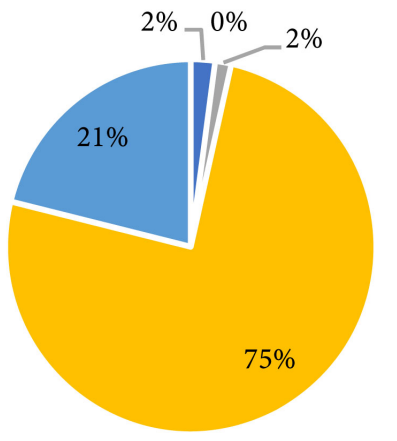

$$
=<2.5=2.5-3.4=3.5-4=4.1-4.4=4.5-5
$$

Figure 1. Percentage of students with various GPAs. 
Table 1. Frequency of the response related to time management on Likert scale.

\begin{tabular}{|c|c|c|c|c|c|}
\hline \multirow{2}{*}{ Questions } & \multicolumn{5}{|c|}{ N (\%) } \\
\hline & 1 & 2 & 3 & 4 & 5 \\
\hline \multicolumn{6}{|c|}{ Student Perception } \\
\hline $\begin{array}{l}\text { Preplanning methods are inefficient, and } \\
\text { it does not affect academic performance. }\end{array}$ & $04(2.8)$ & $13(9.2)$ & $33(23.2)$ & $56(39.4)$ & $36(25.4)$ \\
\hline $\begin{array}{l}\text { My academic performance is degraded } \\
\text { due to misplanning. }\end{array}$ & $18(12.7)$ & $35(24.6)$ & $32(22.5)$ & $39(27.5)$ & $18(12.7)$ \\
\hline $\begin{array}{l}\text { My participating in extracurricular } \\
\text { activities won't affect my academic } \\
\text { performance. }\end{array}$ & $20(14.1)$ & $34(23.9)$ & $36(25.4)$ & $31(21.8)$ & $21(14.8)$ \\
\hline \multicolumn{6}{|c|}{ Positive Attitude } \\
\hline I prefer to manage my time daily. & $36(25.4)$ & $51(35.9)$ & $37(26.1)$ & $12(8.5)$ & $06(4.2)$ \\
\hline I meet the deadline for any work. & $90(63.4)$ & $41(28.9)$ & $04(2.8)$ & $05(3.5)$ & $02(1.4)$ \\
\hline I effectively manage workload. & $25(17.6)$ & $50(35.2)$ & $47(33.1)$ & $12(8.5)$ & $08(5.6)$ \\
\hline $\begin{array}{l}\text { I can adapt and be flexible when changes } \\
\text { occur, reassess priorities, and still } \\
\text { produce quality work. }\end{array}$ & $30(21.1)$ & $57(40.1)$ & $34(23.9)$ & $14(99.9)$ & $07(4.9)$ \\
\hline $\begin{array}{l}\text { I balance between my private time and } \\
\text { study time }\end{array}$ & $21(14.8)$ & $39(27.5)$ & $38(26.8)$ & $33(23.2)$ & $11(7.7)$ \\
\hline $\begin{array}{l}\text { I priorities between various competing } \\
\text { tasks. }\end{array}$ & $43(30.3)$ & $71(50.0)$ & $20(14.1)$ & $07(4.9)$ & $01(0.7)$ \\
\hline
\end{tabular}

Negative attitude

I often tend to delay/postpone my tasks. $\quad 16(11.3) \quad 27(19.0) \quad 21(14.8) \quad 44(31.0) \quad 34(23.9)$

\begin{tabular}{l}
\hline \multicolumn{5}{c}{ Factors with negative impact } \\
$\begin{array}{l}\text { I find this year challenging and it has } \\
\text { affected my time (Online learning). }\end{array}$ \\
$\begin{array}{l}\text { I feel lazy and my study mood is ruined } \\
\text { because of insufficient sleeping-pattern. }\end{array}$
\end{tabular}

Factors with positive impact

I manage stress when handling multiple conflicting duties.

$21(14.8) \quad 37(26.1) \quad 37(26.1) \quad 33(23.2) \quad 14(9.9)$

I have enough time to complete my tasks during the day.

$11(7.7) \quad 31(21.8) \quad 28(19.7) \quad 55(38.7) \quad 17(12.0)$

I usually understand the whole radiology lectures.

$21(14.8) \quad 41(28.9) \quad 23(16.2) \quad 35(24.6) \quad 22(15.5)$

Short term time management

I have a clear established plan for each week's tasks.

$19(13.4) \quad 54(38.0) \quad 35(24.6) \quad 26(18.3) \quad 08(5.6)$

I have a clear established plan for each month's tasks.

$06(4.2) \quad 20(14.1) \quad 36(25.4) \quad 52(36.6) \quad 28(19.7)$ 


\section{Continued}

\begin{tabular}{|c|c|c|c|c|c|}
\hline \multicolumn{6}{|c|}{ Long term time management } \\
\hline $\begin{array}{l}\text { I have a set of goals for the entire } \\
\text { quarter/semester. }\end{array}$ & $12(8.5)$ & $38(26.8)$ & $30(21.1)$ & $44(31.0)$ & $18(12.7)$ \\
\hline $\begin{array}{l}\text { I have a clear established plan } \\
\text { for each year's tasks. }\end{array}$ & $13(9.2)$ & $20(14.1)$ & $31(21.8)$ & $55(38.7)$ & $23(16.2)$ \\
\hline
\end{tabular}

Practices for time management

I feel that my time management skill

needs more improvement (self-assessment).

$47(33.1) \quad 59(41.5) \quad 19(13.4) \quad 12(8.5) \quad 05(3.5)$

I used to put my important dates on a

single calendar (To-do-list program etc.).

$41(28.9) \quad 44(31.0) \quad 20(14.1) \quad 25(17.6) \quad 12(8.5)$

I spend my time wisely and

avoid distractions.

$09(6.3) \quad 39(27.5) \quad 46(32.4) \quad 32(22.5) \quad 16(11.3)$

$1=$ Strongly agree, $2=$ agree, $3=$ Neutral, $4=$ disagree, $5=$ strongly disagree

Table 2. Relationship between student perception and GPA.

\begin{tabular}{|c|c|c|c|c|c|c|c|}
\hline \multirow{2}{*}{$\begin{array}{l}\text { S. } \\
\text { No. }\end{array}$} & & & \multicolumn{4}{|c|}{ GPA } & \multirow{2}{*}{$\begin{array}{c}\text { Pearson } \\
\text { Chi square } \\
\text { p value } \\
\text { (X2, Df) }\end{array}$} \\
\hline & & & $<2.5$ & $3.5-3.9$ & $4-4.5$ & $4.5-5$ & \\
\hline & \multicolumn{7}{|c|}{ Student Perception } \\
\hline \multirow{5}{*}{1} & \multirow{5}{*}{$\begin{array}{l}\text { Preplanning } \\
\text { methods are } \\
\text { inefficient, and } \\
\text { it does not } \\
\text { affect academic } \\
\text { performance. }\end{array}$} & Strongly Agree & $0(0.0)$ & $0(0.0)$ & $3(10.0)$ & $1(0.9)$ & \multirow{5}{*}{$\begin{array}{c}0.190 \\
(16.029,12)\end{array}$} \\
\hline & & Agree & $1(33.3)$ & $0(0.0)$ & $1(3.3)$ & $11(10.3)$ & \\
\hline & & Neutral & $0(0.0)$ & $0(0.0)$ & $11(36.7)$ & $22(20.6)$ & \\
\hline & & Disagree & $1(33.3)$ & $1(50.0)$ & $9(30.0)$ & $45(42.1)$ & \\
\hline & & Strongly Disagree & $1(33.3)$ & $1(50.0)$ & $6(20.0)$ & $28(26.2)$ & \\
\hline \multirow{5}{*}{4} & \multirow{5}{*}{$\begin{array}{l}\text { my academic } \\
\text { performance is } \\
\text { degraded due to } \\
\text { mis planning. }\end{array}$} & Strongly Agree & $0(0.0)$ & $0(0.0)$ & $11(36.7)$ & $7(6.5)$ & \multirow{5}{*}{$\begin{array}{c}0.005 \\
(28.570,12)\end{array}$} \\
\hline & & Agree & $1(33.3)$ & $1(50.0)$ & $9(30.0)$ & $24(22.4)$ & \\
\hline & & Neutral & $0(0.0)$ & $0(0.0)$ & $5(16.7)$ & $27(25.2)$ & \\
\hline & & Disagree & $2(66.7)$ & $1(50.0)$ & $4(13.3)$ & $32(29.9)$ & \\
\hline & & Strongly Disagree & $0(0.0)$ & $0(0.0)$ & $1(3.3)$ & $17(15.9)$ & \\
\hline \multirow{5}{*}{21} & \multirow{5}{*}{$\begin{array}{l}\text { My participating } \\
\text { in extracurricular } \\
\text { activities won't } \\
\text { affect my } \\
\text { academic } \\
\text { performance. }\end{array}$} & Strongly Agree & $0(0.0)$ & $0(0.0)$ & $3(10.0)$ & $17(15.9)$ & \multirow{5}{*}{$\begin{array}{c}0.292 \\
(14.142,12)\end{array}$} \\
\hline & & Agree & $1(33.3)$ & $0(0.0)$ & $6(20.0)$ & $27(25.2)$ & \\
\hline & & Neutral & $0(0.0)$ & $2(100)$ & $5(16.7)$ & $29(27.1)$ & \\
\hline & & Disagree & $1(33.3)$ & $0(0.0)$ & $8(26.7)$ & $22(20.6)$ & \\
\hline & & Strongly Disagree & $1(33.3)$ & $0(0.0)$ & $8(26.7)$ & $12(11.2)$ & \\
\hline & & $y$ agree, $2=$ agree & $3=\mathrm{Ne}$ & $\operatorname{tral}, 4=$ & isagree & $=$ strongl & sagree \\
\hline
\end{tabular}


Table 3. Relationship between behavior for time and GPA of last year.

\begin{tabular}{|c|c|c|c|c|c|c|c|}
\hline \multirow{2}{*}{ S. No. } & \multirow{2}{*}{\multicolumn{2}{|c|}{ Behavior for time management }} & \multicolumn{4}{|c|}{ GPA } & \multirow{2}{*}{$\begin{array}{c}\text { Pearson } \\
\text { Chi square } \\
\text { p value } \\
\text { (X2, Df) }\end{array}$} \\
\hline & & & $<2.5$ & $3.5-3.9$ & $4-4.5$ & $4.5-5$ & \\
\hline & \multicolumn{7}{|c|}{ Positive Attitude } \\
\hline \multirow{5}{*}{5} & \multirow{5}{*}{$\begin{array}{l}\text { I prefer to manage } \\
\text { my time daily. }\end{array}$} & Strongly Agree & $0(0.0)$ & $0(0.0)$ & $5(16.7)$ & $31(29.0)$ & \multirow{5}{*}{$\begin{array}{c}0.276 \\
(14.399,12)\end{array}$} \\
\hline & & Agree & $1(33.3)$ & $2(100)$ & $15(50.0)$ & $33(30.8)$ & \\
\hline & & Neutral & $2(66.7)$ & $0(0.0)$ & $9(30.0)$ & $26(24.3)$ & \\
\hline & & Disagree & $0(0.0)$ & $0(0.0)$ & $0(0.0)$ & $12(11.2)$ & \\
\hline & & Strongly Disagree & $0(0.0)$ & $0(0.0)$ & $1(3.3)$ & $5(4.7)$ & \\
\hline \multirow{5}{*}{13} & \multirow{5}{*}{$\begin{array}{l}\text { I meet the deadline } \\
\text { for any work. }\end{array}$} & Strongly Agree & $2(66.7)$ & $0(0.0)$ & $14(46.7)$ & $74(69.2)$ & \multirow{5}{*}{$\begin{array}{c}0.005 \\
(28.215,12)\end{array}$} \\
\hline & & Agree & $1(33.3)$ & $1(50.0)$ & $15(50.0)$ & $24(22.4)$ & \\
\hline & & Neutral & $0(0.0)$ & $1(50.0)$ & $1(3.3)$ & $2(1.9)$ & \\
\hline & & Disagree & $0(0.0)$ & $0(0.0)$ & $0(0.0)$ & $5(4.7)$ & \\
\hline & & Strongly Disagree & $0(0.0)$ & $0(0.0)$ & $0(0.0)$ & $2(1.9)$ & \\
\hline \multirow{5}{*}{15} & \multirow{5}{*}{$\begin{array}{c}\text { I effectively } \\
\text { manage workload. }\end{array}$} & Strongly Agree & $0(0.0)$ & $0(0.0)$ & $4(13.3)$ & $21(19.6)$ & \multirow{5}{*}{$\begin{array}{c}0.142 \\
(17.214,12)\end{array}$} \\
\hline & & Agree & $0(0.0)$ & $0(0.0)$ & $10(33.3)$ & $40(37.4)$ & \\
\hline & & Neutral & $3(100)$ & $1(50.0)$ & $8(26.77)$ & $35(32.7)$ & \\
\hline & & Disagree & $0(0.0)$ & $1(50.0)$ & $5(16.7)$ & $6(5.6)$ & \\
\hline & & Strongly Disagree & $0(0.0)$ & $0(0.0)$ & $3(10.0)$ & $5(4.7)$ & \\
\hline \multirow{5}{*}{20} & \multirow{5}{*}{$\begin{array}{l}\text { I can adapt and be } \\
\text { flexible when } \\
\text { changes occur, } \\
\text { reassess priorities, } \\
\text { and still produce } \\
\text { quality work. }\end{array}$} & Strongly Agree & $0(0.0)$ & $0(0.0)$ & $6(20.0)$ & $24(22.4)$ & \multirow{5}{*}{$\begin{array}{c}0.881 \\
(6.629,12)\end{array}$} \\
\hline & & Agree & $1(33.3)$ & $1(50.0)$ & $10(33.3)$ & $45(42.1)$ & \\
\hline & & Neutral & $1(33.3)$ & $1(50.0)$ & $10(33.3)$ & $22(20.6)$ & \\
\hline & & Disagree & $1(33.3)$ & $0(0.0)$ & $2(6.7)$ & $11(10.3)$ & \\
\hline & & Strongly Disagree & $0(0.0)$ & $0(0.0)$ & $2(6.7)$ & $5(4.7)$ & \\
\hline \multirow{5}{*}{22} & \multirow{5}{*}{$\begin{array}{l}\text { I balance between } \\
\text { my private time } \\
\text { and study time }\end{array}$} & Strongly Agree & $0(0.0)$ & $0(0.0)$ & $2(6.7)$ & $19(17.8)$ & \multirow{5}{*}{$\begin{array}{c}0.502 \\
(11.317,12)\end{array}$} \\
\hline & & Agree & $0(0.0)$ & $1(50.0)$ & $6(20.0)$ & $32(29.9)$ & \\
\hline & & Neutral & $2(66.7)$ & $1(50.0)$ & $9(30.0)$ & $26(24.3)$ & \\
\hline & & Disagree & $1(33.3)$ & $0(0.0)$ & $11(36.7)$ & $21(19.6)$ & \\
\hline & & Strongly Disagree & $0(0.0)$ & $0(0.0)$ & $2(6.7)$ & $9(8.4)$ & \\
\hline \multirow{6}{*}{17} & \multirow{5}{*}{$\begin{array}{c}\text { I priorities } \\
\text { between various } \\
\text { competing tasks. }\end{array}$} & Strongly Agree & $1(33.3)$ & $1(50.0)$ & $6(20.0)$ & $35(32.7)$ & \multirow{5}{*}{$\begin{array}{c}0.982 \\
(4.058,12)\end{array}$} \\
\hline & & Agree & $1(33.3)$ & $1(50.0)$ & $17(56.7)$ & $52(48.6)$ & \\
\hline & & Neutral & $1(33.3)$ & $0(0.0)$ & $5(16.7)$ & $14(13.1)$ & \\
\hline & & Disagree & $0(0.0)$ & $0(0.0)$ & $2(6.7)$ & $5(4.7)$ & \\
\hline & & Strongly Disagree & $0(0.0)$ & $0(0.0)$ & $0(0.0)$ & $1(0.9)$ & \\
\hline & \multicolumn{7}{|c|}{ Negative attitude } \\
\hline \multirow{5}{*}{14} & \multirow{5}{*}{$\begin{array}{l}\text { I often tend to } \\
\text { delay/postpone } \\
\text { my tasks. }\end{array}$} & Strongly Agree & $1(33.3)$ & $0(0.0)$ & $10(33.3)$ & $5(4.7)$ & \\
\hline & & Agree & $0(0.0)$ & $1(50.0)$ & $7(23.3)$ & $19(17.8)$ & \\
\hline & & Neutral & $1(33.3)$ & $0(0.0)$ & $4(13.3)$ & $16(15.0)$ & \\
\hline & & Disagree & $0(0.0)$ & $0(0.0)$ & $7(23.3)$ & $35(32.70$ & \\
\hline & & Strongly Disagree & $1(33.3)$ & $1(50.0)$ & $2(6.7)$ & $32(29.9)$ & \\
\hline
\end{tabular}




\section{Continued}

\begin{tabular}{|c|c|c|c|c|c|c|c|}
\hline \multicolumn{8}{|c|}{ Short term time management } \\
\hline \multirow{5}{*}{6} & \multirow{5}{*}{$\begin{array}{l}\text { I have a clear } \\
\text { established plan } \\
\text { for each } \\
\text { week's tasks. }\end{array}$} & Strongly Agree & $0(0.0)$ & $1(50.0)$ & $4(13.3)$ & $14(13.1)$ & \multirow{5}{*}{$\begin{array}{c}0.833 \\
(7.359,12)\end{array}$} \\
\hline & & Agree & $1(33.3)$ & $0(0.0)$ & $9(30.0)$ & $44(41.1)$ & \\
\hline & & Neutral & $1(33.3)$ & $1(50.0)$ & $7(23.3)$ & $26(24.3)$ & \\
\hline & & Disagree & $1(33.3)$ & $0(0.0)$ & $7(23.3)$ & $18(16.8)$ & \\
\hline & & Strongly Disagree & $0(0.0)$ & $0(0.0)$ & $3(10.0)$ & $5(4.7)$ & \\
\hline \multirow{6}{*}{7} & \multirow{5}{*}{$\begin{array}{l}\text { I have a clear } \\
\text { established plan } \\
\text { for each } \\
\text { month's tasks. }\end{array}$} & Strongly Agree & $0(0.0)$ & $0(0.0)$ & $2(6.7)$ & $4(3.7)$ & \multirow{5}{*}{$\begin{array}{c}0.486 \\
(11.510,12)\end{array}$} \\
\hline & & Agree & $0(0.0)$ & $0(0.0)$ & $6(20.0)$ & $14(13.1)$ & \\
\hline & & Neutral & $2(66.7)$ & $2(100)$ & $6(20.0)$ & $26(24.3)$ & \\
\hline & & Disagree & $1(33.3)$ & $0(0.0)$ & $9(30.0)$ & $42(39.3)$ & \\
\hline & & Strongly Disagree & $0(0.0)$ & $0(0.0)$ & $7(23.3)$ & $21(19.6)$ & \\
\hline & \multicolumn{7}{|c|}{ Long term time management } \\
\hline \multirow{5}{*}{8} & \multirow{5}{*}{$\begin{array}{l}\text { I have a set of } \\
\text { goals for the entire } \\
\text { quarter/semester. }\end{array}$} & Strongly Agree & $0(0.0)$ & $0(0.0)$ & $3(10.0)$ & $9(8.4)$ & \multirow{5}{*}{$\begin{array}{c}0.903 \\
(6.254,12)\end{array}$} \\
\hline & & Agree & $1(33.3)$ & $1(50.0)$ & $8(26.7)$ & $28(26.2)$ & \\
\hline & & Neutral & $0(0.0)$ & $0(0.0)$ & $8(26.7)$ & $22(20.6)$ & \\
\hline & & Disagree & $2(66.7)$ & $1(50.0)$ & $6(20.0)$ & $35(32.7)$ & \\
\hline & & Strongly Disagree & $0(0.0)$ & $0(0.0)$ & $5(16.7)$ & $13(12.1)$ & \\
\hline \multirow{5}{*}{9} & \multirow{5}{*}{$\begin{array}{c}\text { I have a clear } \\
\text { established plan } \\
\text { for each year's tasks. }\end{array}$} & Strongly Agree & $1(33.3)$ & $0(0.0)$ & $2(6.7)$ & $10(9.3)$ & \multirow{5}{*}{$\begin{array}{c}0.168 \\
(16.544,12)\end{array}$} \\
\hline & & Agree & $0(0.0)$ & $1(50.0)$ & $2(6.7)$ & $17(15.9)$ & \\
\hline & & Neutral & $0(0.0)$ & $0(0.0)$ & $11(36.7)$ & $20(18.7)$ & \\
\hline & & Disagree & $2(66.7)$ & $1(50.0)$ & $7(23.3)$ & $45(42.1)$ & \\
\hline & & Strongly Disagree & $0(0.0)$ & $0(0.0)$ & $8(26.7)$ & $15(14.0)$ & \\
\hline
\end{tabular}

Table 4. Relationship between various factors of time management and GPA.

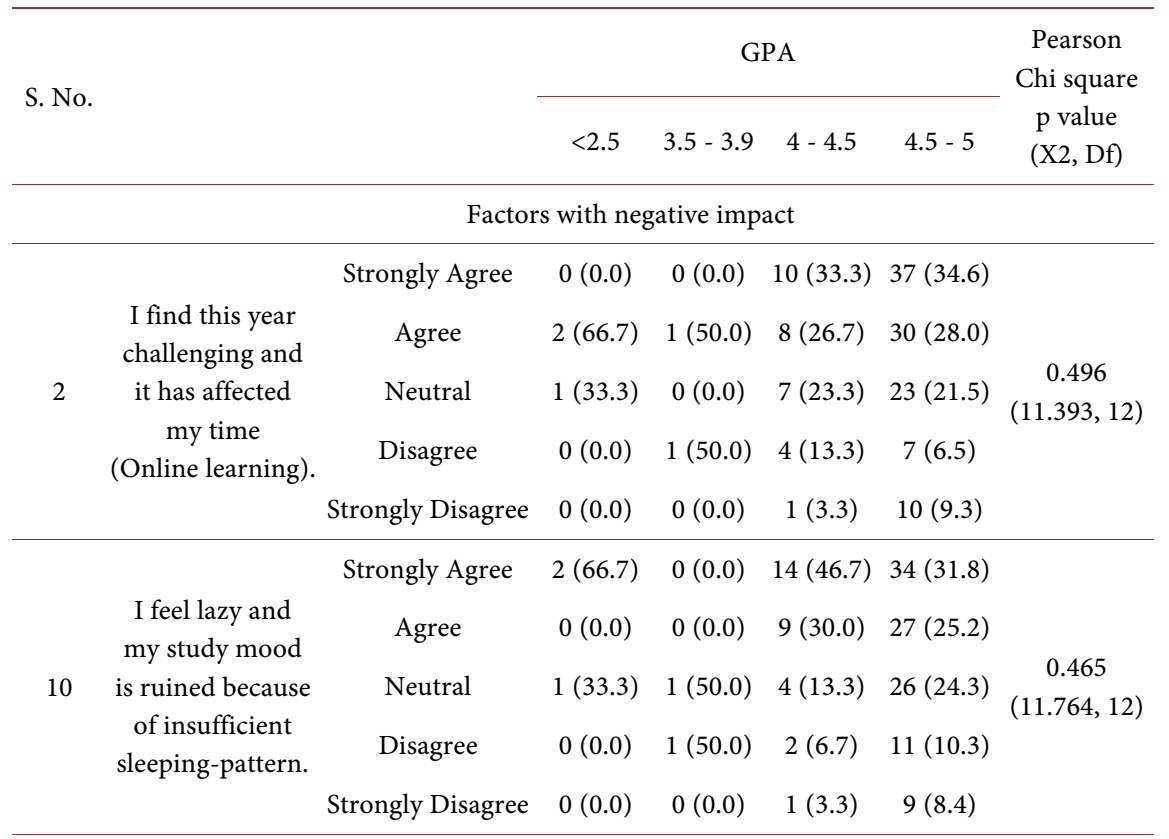




\section{Continued}

\begin{tabular}{|c|c|c|c|c|c|c|c|}
\hline \multicolumn{8}{|c|}{ Factors with positive impact } \\
\hline \multirow{5}{*}{3} & \multirow{5}{*}{$\begin{array}{l}\text { I manage stress } \\
\text { when handling } \\
\text { multiple } \\
\text { conflicting duties. }\end{array}$} & Strongly Agree & $0(0.0)$ & $0(0.0)$ & $5(16.7)$ & $16(15.0)$ & \multirow{5}{*}{$\begin{array}{c}0.611 \\
(10.055,12)\end{array}$} \\
\hline & & Agree & $0(0.0)$ & $1(50.0)$ & $6(20.0)$ & $30(28.0)$ & \\
\hline & & Neutral & $2(66.7)$ & $1(50.0)$ & $5(16.7)$ & $29(27.1)$ & \\
\hline & & Disagree & $1(33.3)$ & $0(0.0)$ & $11(36.7)$ & $21(19.6)$ & \\
\hline & & Strongly Disagree & $0(0.0)$ & $0(0.0)$ & $3(10.0)$ & $11(10.3)$ & \\
\hline \multirow{5}{*}{11} & \multirow{5}{*}{$\begin{array}{l}\text { I have enough } \\
\text { time to complete } \\
\text { my tasks during } \\
\text { the day. }\end{array}$} & & $0(0.0)$ & $0(0.0)$ & $3(10.0)$ & $8(7.5)$ & \multirow{5}{*}{$\begin{array}{c}0.962 \\
(4.869,12)\end{array}$} \\
\hline & & $\begin{array}{l}\text { Strongly Agree } \\
\text { Agree }\end{array}$ & $1(33.3)$ & $1(50.0)$ & $6(20.0)$ & $23(21.5)$ & \\
\hline & & Neutral & $1(33.3)$ & $1(50.0)$ & $7(23.3)$ & $19(17.8)$ & \\
\hline & & $\begin{array}{l}\text { Disagree } \\
\text { Strongly Disagree }\end{array}$ & $1(33.3)$ & $0(0.0)$ & $11(36.7)$ & $43(40.2)$ & \\
\hline & & & $0(0.0)$ & $0(0.0)$ & $3(10.0)$ & $14(13.1)$ & \\
\hline \multirow{6}{*}{12} & \multirow{5}{*}{$\begin{array}{c}\text { understand the } \\
\text { whole radiology } \\
\text { lectures. }\end{array}$} & Strongly Agree & $0(0.0)$ & $0(0.0)$ & $7(23.3)$ & $14(13.1)$ & \multirow{5}{*}{$\begin{array}{c}0.452 \\
(11.921,12)\end{array}$} \\
\hline & & Agree & $0(0.0)$ & $0(0.0)$ & $9(30.0)$ & $32(29.9)$ & \\
\hline & & Neutral & $0(0.0)$ & $1(50.0)$ & $6(20.0)$ & $16(20.0)$ & \\
\hline & & Disagree & $2(66.7)$ & $1(50.0)$ & $6(20.0)$ & $26(24.3)$ & \\
\hline & & Strongly Disagree & $1(33.3)$ & $0(0.0)$ & $2(6.7)$ & $19(17.8)$ & \\
\hline & \multicolumn{7}{|c|}{ Practices for time management } \\
\hline \multirow{5}{*}{16} & \multirow{5}{*}{$\begin{array}{l}\text { I feel that my } \\
\text { time } \\
\text { management } \\
\text { skill needs more } \\
\text { improvement } \\
\text { (self-assessment). }\end{array}$} & Strongly Agree & $1(33.3)$ & $0(0.0)$ & $12(40.0)$ & $34(31.8)$ & \multirow{5}{*}{$\begin{array}{c}0.478 \\
(11.601,12)\end{array}$} \\
\hline & & Agree & $2(66.7)$ & $1(50.0)$ & $14(46.7)$ & $42(39.3)$ & \\
\hline & & Neutral & $0(0.0)$ & $0(0.0)$ & $4(13.3)$ & $15(14.0)$ & \\
\hline & & Disagree & $0(0.0)$ & $1(50.0)$ & $0(0.0)$ & $11(10.3)$ & \\
\hline & & Strongly Disagree & $0(0.0)$ & $0(0.0)$ & $0(0.0)$ & $5(4.7)$ & \\
\hline \multirow{5}{*}{18} & \multirow{5}{*}{$\begin{array}{c}\text { I used to put } \\
\text { my important } \\
\text { dates on a single } \\
\text { calendar } \\
\text { (To-do-list } \\
\text { program etc.). }\end{array}$} & Strongly Agree & $0(0.0)$ & $2(100)$ & $3(10.0)$ & $36(33.6)$ & \multirow{5}{*}{$\begin{array}{c}0.047 \\
(21.264,12)\end{array}$} \\
\hline & & Agree & $1(33.3)$ & $0(0.0)$ & $8(26.7)$ & $35(32.7)$ & \\
\hline & & Neutral & $0(0.0)$ & $0(0.0)$ & $6(20.0)$ & $14(13.1)$ & \\
\hline & & Disagree & $1(33.3)$ & $0(0.0)$ & $8(26.7)$ & $15(14.0)$ & \\
\hline & & Strongly Disagree & $0(0.0)$ & $0(0.0)$ & $5(16.7)$ & $7(6.5)$ & \\
\hline \multirow{5}{*}{19} & \multirow{5}{*}{$\begin{array}{l}\text { I spend my } \\
\text { time wisely } \\
\text { and avoid } \\
\text { distractions. }\end{array}$} & Strongly Agree & $0(0.0)$ & $0(0.0)$ & $1(3.3)$ & $8(7.5)$ & \multirow{5}{*}{$\begin{array}{c}0.862 \\
(6.937,12)\end{array}$} \\
\hline & & Agree & $0(0.0)$ & $1(50.0)$ & $8(26.7)$ & $30(28.0)$ & \\
\hline & & Neutral & $2(66.7)$ & $0(0.0)$ & $8(26.7)$ & $36(33.6)$ & \\
\hline & & Disagree & $1(33.3)$ & $1(50.0)$ & $8(26.7)$ & $22(20.6)$ & \\
\hline & & Strongly Disagree & $0(0.0)$ & $0(0.0)$ & $5(16.7)$ & $11(10.3)$ & \\
\hline
\end{tabular}

\section{Discussion}

This study aimed to find the impact of time management on the academic performance of students among the diagnostic radiology technology students at KAU. There were more female students that answered the questionnaire. Ac- 
cording to student's perception, preplanning their studies had been beneficial for their academic performance. However, less than half of the students agreed that they manage their time, which could be due to various reasons. In this study students agreed that insufficient sleep pattern makes their feel lazy and they are unable to manage time. Furthermore, majority also believed that the last year had been challenging (due to covid-19 pandemic) and affected their time (and online learning). Previous study on undergraduate nursing students also found that decreasing procrastination and increasing time management will help the students to have better academic performance, less anxiety and will be better prepared for working in hospital (Romero-Blanco et al., 2020). Similar to the results of this stud, previous study Romero-Blanco et al. (2020) also posited that during previous year of covid-19 pandemic, sleep pattern among students has been affected. Elagra et al. (2016) suggest that decrease in quality sleep also decreases the academic performance in the students.

The students with positive attitude and who claimed to manage time and meet their deadlines were also had high academic grades. Whereas, short-term and long-term time management were not associated with the high GPA. Contrast to it, previous study had resulted time management behavior such as short range and long-range management to be effective (Ganguly, Kulkarni, \& Gupta, 2017). However, those studies are old and suggested effect in mature female students only. Therefore, a longitudinal study is required to observe the relationship between time management behavior and academic achievement for a definite relationship.

Among the high GPA holders, majority of them agreed to make a diary, to-do list or put important dates on the calendar. It might be due to the established relationship between maintaining a task list with effective time management (Caligor, 2017). However, according to recent study (Evans, 2017), it is important to digitalize this to-do list or task list for better accessibility, timely reminders and collaborations. This study is limited based on its design being a cross-sectional survey which is a snapshot of a situation. Therefore, for better management, it is important to have a longitudinal prospective study with long term follow up throughout the academic years to further analyze the relationship. It is also recommended for students to maintain their sleeping pattern for better time management. Furthermore, as a to-do list or making task list on calendar has been a positive factor in time management and academic achievement, it is recommended to use digitalized to-do lists for better performance.

In conclusion, according to student's perception, preplanning their studies had been beneficial for their academic performance. However, less than half of the students agreed that they manage their time. No relationship was found between short range and long-range time management behavior on academic achievement. This study recommends better sleeping pattern and making of to-do list among students for better time management, which could lead to high academic achievement. 


\section{Acknowledgements}

The author is very thankful to all the associated personnel and departments in any reference that contributed in/for the purpose of this research.

\section{Conflicts of Interest}

The authors report no financial or other relationship relevant to the subject of this article.

\section{Funding Sources}

No financial support was received for this study.

\section{Ethical Considerations}

This study has been reviewed and approved by the Faculty of Applied Medical Sciences, King Abdul-Aziz University.

\section{References}

Adams, R. V., \& Blair, E. (2019). Impact of Time Management Behaviors on Undergraduate Engineering Students' Performance. SAGE Open, 9, 1-11. https://doi.org/10.1177/2158244018824506

Aeon, B., \& Aguinis, H. (2017). It's about Time: New Perspectives and Insights on Time Management. Academy of Management Perspectives, 31, 309-330. https://doi.org/10.5465/amp.2016.0166

Ahmad, S., \& Ch, H. (2017). Relationship of Classroom Management Strategies with Academic Performance of Students at College Level. Bulletin of Education and Research, 39, 239-249.

Broadbent, J. (2017). Comparing Online and Blended Learner's Self-Regulated Learning Strategies and Academic Performance. The Internet and Higher Education, 33, 24-32. https://doi.org/10.1016/j.iheduc.2017.01.004

Caligor, D. (2017). U.S. Patent No. 9,659,260. Washington DC: U.S. Patent and Trademark Office.

Claessens, B. J., Van Eerde, W., Rutte, C. G., \& Roe, R. A. (2004). Planning Behavior and Perceived Control of Time at Work. Journal of Organizational Behavior, 25, 937-950. https://doi.org/10.1002/job.292

Elagra, M. I., Rayyan, M. R., Alnemer, O. A., Alshehri, M. S., Alsaffar, N. S., Al-Habib, R. S., \& Almosajen, Z. A. (2016). Sleep Quality among Dental Students and Its Association with Academic Performance. Journal of International Society of Preventive \& Community Dentistry, 6, 296. https://doi.org/10.4103/2231-0762.186788

Evans, R. S. (2017). Digitize Your To-Do List.

Ganguly, S., Kulkarni, M., \& Gupta, M. (2017). Predictors of Academic Performance among Indian Students. Social Psychology of Education, 20, 139-157. https://doi.org/10.1007/s11218-016-9345-y

Gupta, D., \& Chitkara, S. (2018). Effect of Time Management on Academic Performance of Management Students. Global Journal on Recent Advancement in Business Forecasting and Marketing Intelligence, 2, 1-14.

Jenaabadi, H., Nastiezaie, N., \& Jalalzaei, S. (2016). The Effect of Time Management 
Training on Student's Test Anxiety. Journal of Nursing Education, 5, 12-22.

Kearns, H., \& Gardiner, M. (2007). Is It Time Well Spent? The Relationship between Time Management Behaviours, Perceived Effectiveness and Work-Related Morale and Distress in a University Context. High Education Research \& Development, 26, 235 247. https://doi.org/10.1080/07294360701310839

Kelly, W. E. (2002). Harnessing the River of Time: A Theoretical Framework of Time Use Efficiency with Suggestions for Counselors. Journal of Employment Counseling, 39, 12-21. https://doi.org/10.1002/j.2161-1920.2002.tb00504.x

Kharadze, N., Gulua, E., \& Davit, D. (2017). Free-Time Management among Master's Degree Students of Georgia. European Journal of Social Science Education and Research, 4, 24-33. https://doi.org/10.26417/ejser.v11i2.p24-33

Krause, K. L., \& Coates, H. (2008). Students' Engagement in First-Year University. Assessment \& Evaluation in Higher Education, 33, 493-505. https://doi.org/10.1080/02602930701698892

Kumar, P. M., \& Aithal, P. S. (2019). Importance of Time as Resource in Managing Organizations. Proceedings of National Conference on Recent Advances in Technological Innovations in IT, Management, Education \& Social Sciences, ISBN No: 978-81941751-6-2, October 2019, 45-52. https://ssrn.com/abstract $=3513000$

McKenzie, K., Gow, K., \& Schweitzer, R. (2004). Exploring First-Year Academic Achievement through Structural Equation Modelling. Higher Education Research \& Development, 23, 95-112. https://doi.org/10.1080/0729436032000168513

Nadinloyi, K. B., Hajloo, N., Garamaleki, N. S., \& Sadeghi, H. (2013). The Study Efficacy of Time Management Training on Increases Academic Time Management of Students. Procedia-Social and Behavioral Sciences, 84, 134-138. https://doi.org/10.1016/j.sbspro.2013.06.523

Nasrullah, S., \& Khan, M. S. (2015). The Impact of Time Management on the Students' Academic Achievements.

Nayak, S. G. (2019). Impact of Procrastination and Time-Management on Academic Stress among Undergraduate Nursing Students: A Cross Sectional Study. International Journal of Caring Sciences, 12, 1480-1486.

Nieuwoudt, J. E., \& Brickhill, M. (2017). Time Management and Attitude towards Science as Predictors of Academic Success in an Enabling Science Subject: A Preliminary EXploratory Study. National Association of Enabling Educators of Australia (NAEEA).

Pugh, C. M., \& Nathwani, J. N. (2017). Time Management. In Success in Academic Surgery (pp. 187-199). Cham: Springer. https://doi.org/10.1007/978-3-319-43952-5 15

Razali, S. N. A. M., Rusiman, M. S., Gan, W. S., \& Arbin, N. (2018). The Impact of Time Management on Students' Academic Achievement. Journal of Physics: Conference Series, 995, Article ID: 012042. https://doi.org/10.1088/1742-6596/995/1/012042

Romero-Blanco, C., Rodríguez-Almagro, J., Onieva-Zafra, M. D., Parra-Fernández, M. L., Prado-Laguna, M. D. C., \& Hernández-Martínez, A. (2020). Sleep Pattern Changes in Nursing Students during the COVID-19 Lockdown. International Journal of Environmental Research and Public Health, 17, 5222. https://doi.org/10.3390/ijerph17145222

Savino, D. M. (2016). Frederick Winslow Taylor and His Lasting Legacy of Functional Leadership Competence. Journal of Leadership, Accountability and Ethics, 13, 70.

Sayari, K., Jalagat, R., \& Dalluay, V. (2017). Assessing the Relationship of Time Management and Academic Performance of the Business Students in Al-Zahra College for Women. European Business \& Management, 3, 1-8. https://doi.org/10.11648/j.ebm.20170301.11 
Scherer, S., Talley, C. P., \& Fife, J. E. (2017). How Personal Factors Influence Academic Behavior and GPA in African American STEM Students. SAGE Open, 7, 21582440 17704686. https://doi.org/10.1177/2158244017704686

Sharma, S., \& Would, I. (2004). Time Management among Dental Students-Something to Consider? Stress, 1, 100-101.

https://www.dentaltown.com/images/Dentaltown/magimages/0417/ukStudentTime.pdf

Valle, A., Regueiro, B., Núñez, J. C., Rodríguez, S., Piñeiro, I., \& Rosário, P. (2016). Academic Goals, Student Homework Engagement, and Academic Achievement in Elementary School. Frontiers in Psychology, 7, 463.

https://doi.org/10.3389/fpsyg.2016.00463

Wang, W. C., Kao, C. H., Huan, T. C., \& Wu, C. C. (2011). Free Time Management Contributes to Better Quality of Life: A Study of Undergraduate Students in Taiwan. Journal of Happiness Studies, 12, 561-573. https://doi.org/10.1007/s10902-010-9217-7 


\section{Appendix}

\section{Research questionnaire}

Statement Questions (in a scale of 5 choose from strongly disagree - Strongly agree)

1) Preplanning methods are inefficient, and it does not affect academic performance.

2) I find this year challenging and it has affected my time (Online learning).

3) I manage stress when handling multiple conflicting duties.

4) My academic performance is degraded due to mis planning.

5) I prefer to manage my time daily.

6) I have a clear established plan for each week's tasks.

7) I have a clear established plan for each month's tasks.

8) I have a set of goals for the entire quarter/semester.

9) I have a clear established plan for each year's tasks.

10) I feel lazy and my study mood is ruined because of insufficient sleeping-pattern.

11) I have enough time to complete my tasks during the day.

12) I usually understand the whole radiology lectures.

13) I meet the deadline for any work.

14) I often tend to delay/postpone my tasks.

15) I effectively manage workload.

16) I feel that my time management skill needs more improvement (self-assessment).

17) I priorities between various competing tasks.

18) I used to put my important dates on a single calendar (To-do-list program etc.).

19) I spend my time wisely and avoid distractions.

20) I can adapt and be flexible when changes occur, reassess priorities, and still produce quality work.

21) My participating in extracurricular activities won't affect my academic performance.

22) I balance between my private time and study time

23) Please specify your last year GPA $(\sqrt{ })$ :

a) I achieve a (4.5 - 5) last year

b) I achieve a (4.0 - 4.4) last year

c) I achieve a 3.5 - 3.9 last year

d) I achieve a 2.5 - 3.4 last year

e) I achieve less than (2.5) last year 